\title{
Studies with 2-arylhydrazononitriles: a new convenient synthesis of 2, 4-disubstituted-1,2,3-triazole-5-amines
}

\author{
Said Ahmed Soliman Ghozlan ${ }^{\mathrm{a} *}$, Ismail Abdelshafy Abdelhamid ${ }^{\mathrm{a} *}$, \\ Hamada Mohamed Ibrahim, ${ }^{b}$ and Mohamed Hilmy Elnagdi ${ }^{a}$
}

${ }^{a}$ Department of Chemistry, Faculty of Science, Cairo University, Giza, A. R. Egypt

${ }^{b}$ Department of Chemistry, Faculty of Science, Fayoum University, A. R. Egypt

E-mail: s_ghozlan@yahoo.com; ismail_shafy@yahoo.com

\begin{abstract}
A new simple and efficient approach to 2,4-disubstituted 1,2,3-triazoles-5-amines from the reaction of 2-arylhydrazononitriles and hydroxylamine is described. Investigation of behavior of 3-phenyl-3oxo-2-arylhydrazonopropane nitriles has been undertaken. In addition to readily reported formation of aminoisoxazoles, 4-benzoyl-1-aryl-1,2,3-triazole-5-amines 5f,g were prepared via cyclising products of reacting $\mathbf{2 f}, \mathbf{g}$ with hydroxylamine in basic medium.
\end{abstract}

Keywords: Hydrazononitriles, 1,2,3-triazoles, aminoisoxazoles

\section{Results and Discussion}

1,2,3-triazoles constitute a class of compounds which have attracted considerable attention in industry, agricultural and medicinal chemistry ${ }^{[1-4]}$. Owing to their importance an impressive number of work has been published on the chemistry and synthesis of 1,2,3-triazoles ${ }^{[5-16]}$. However, literature inspection indicated lack of efficient synthetic approach to 2-substituted1,2,3-triazoleamines ${ }^{[15]}$. Cyclization of 2-arylhydrazonooximes into 1,2,3-triazole oxides has been reported some time ago ${ }^{[15]}$. Moreover the acetylated derivative of Mesoaldehyde 1,3dioxime-2-arylhydrazone has been reported to cyclise in acetic acid in presence of $\mathrm{CsCO}_{3}$ into 1,2,3-triazole ${ }^{[17]}$. We have looked into possible utility of a modification of this approach as a route for synthesis of amino-1,2,3-triazoles. Thus the arylhydrazononitriles 2a-g were prepared via coupling appropriate active methylene nitriles $\mathbf{1}$ with aromatic diazonium salts. Compound 2e has been prepared via reacting $\mathbf{3}$ with aryldiazonium salts as has been recently described ${ }^{[18]}$ (cf. scheme 1). 


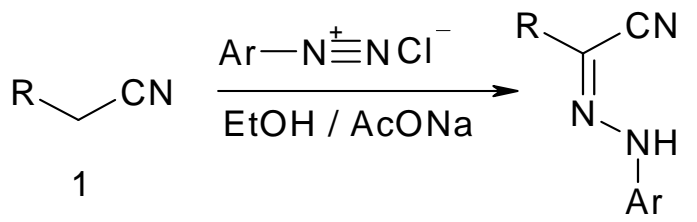

$$
\begin{aligned}
\text { 2a, } \mathrm{R}=\text { benzimidazol-2-yl; } & \mathrm{Ar}=\mathrm{C}_{6} \mathrm{H}_{5} \\
\text { b, } \mathrm{R}=\text { benzimidazol-2-yl; } & \mathrm{Ar}=\mathrm{C}_{6} \mathrm{H}_{4} \mathrm{Cl}(\mathrm{p}) \\
\text { c, } \mathrm{R}=\text { benzthiazol-2-yl; } & \mathrm{Ar}=\mathrm{C}_{6} \mathrm{H}_{5} \\
\text { d, } \mathrm{R}=\text { benzthiazol-2-yl; } & \mathrm{Ar}=\mathrm{C}_{6} \mathrm{H}_{4} \mathrm{Cl}(\mathrm{p}) \\
\mathrm{e}, \mathrm{R}=\mathrm{C}_{6} \mathrm{H}_{5} ; & \mathrm{Ar}=\mathrm{C}_{6} \mathrm{H}_{4} \mathrm{C}(\mathrm{p}) \mathrm{I} \\
\mathrm{f}, \mathrm{R}=\mathrm{COPh} ; & \mathrm{Ar}=\mathrm{C}_{6} \mathrm{H}_{5} \\
\text { g, R }=\text { COPh; } & \mathrm{Ar}=\mathrm{C}_{6} \mathrm{H}_{4} \mathrm{Cl}(\mathrm{p})
\end{aligned}
$$

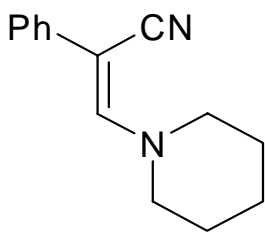

3

\section{Scheme 1}

The arylhydrazones 2a-e reacted smoothly with hydroxylamine hydrochloride in ethanol/sodium acetate to afford amidooximes 4a-e in excellent yields (cf. scheme 2). The ${ }^{13} \mathrm{C}$ NMR of compounds 4c,d revealed expected 13 carbon signals. However ${ }^{1} \mathrm{H}$ NMR revealed expected two signals for an oxime $\mathrm{OH}$ with the relative intensities of $1: 1.2$, other two signals for hydrazone $\mathrm{NH}$ and amino protons appear also at two different positions. This may be interpreted by assuming that amidooximes 4c,d exists at least in DMSO as a mixture of 4c,d (I) and 4c,d (II) (cf. fig. 1). However, when 4a-e were refluxed in DMF in presence of anhydrous sodium acetate 1,2,3-triazoles 5a-e were obtained in 75-85\% yields (cf. scheme 2). Compounds 5a-e were directly obtained from reaction of 2a-e with hydroxylamine hydrochloride in refluxing DMF in presence of anhydrous sodium acetate.

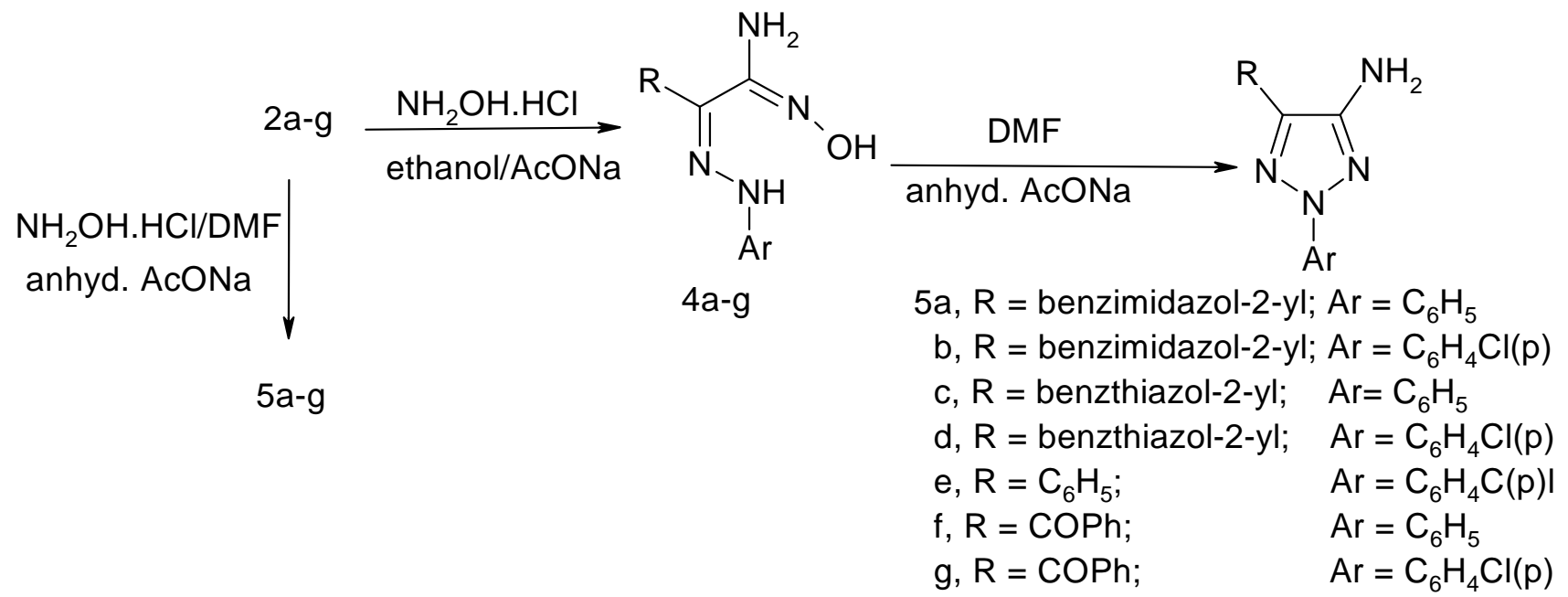

\section{Scheme 2}




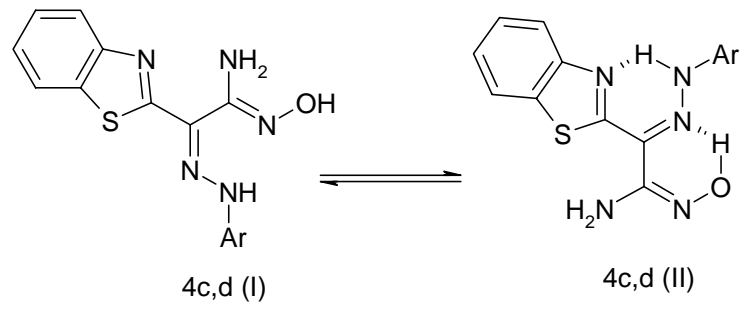

Figure 1. Stereoisomeric conformers of compounds 4c,d.

In accordance with literature ${ }^{[19]}$ compound $\mathbf{2 f}$ reacted with hydroxylamine hydrochloride in ethanol/sodium acetate solution to yield amidooxime $\mathbf{4 f}$ which then cyclised smoothly via loss of water in DMF and in presence of anhydrous sodium acetate. At first we thought that this product is then the arylazoisoxazole 6. However, physical data of the reaction product were found different from those reported in literature ${ }^{[19]}$ for $\mathbf{6}$. Compound $\mathbf{6}$ has been prepared from cyclization of amidooxime $4 \mathrm{f}$ in Conc. $\mathrm{H}_{2} \mathrm{SO}_{4}$ and was found to be really different than our cyclization product $\mathbf{5 f}$ (cf. scheme 3 ). However at this stage it seemed quite probable that may be our cyclization product is that isoxazole and literature product is really the 1,2,3-triazole. This possibility seemed in fact however, least likely as ${ }^{13} \mathrm{C}$ NMR of cyclization product in DMF revealed carbonyl carbon at $\delta 185.99 \mathrm{ppm}$. Conclusive evidence for structure has been obtained via inspection of X-ray structure of compound $5 \mathrm{~g}$ (C.f. fig 2 and table 1) for bond angles and bond lengths. X-ray data ${ }^{[20]}$ clearly indicate that the triazole ring is almost planer with extensive delocalization of nitrogen lone pair $\mathrm{N}-2$ at exocyclic carbonyl function such resonance stabilization may be behind readily formation of the triazole ring in this case. Such delocalization can also be envisioned for other derivatives of 5 .

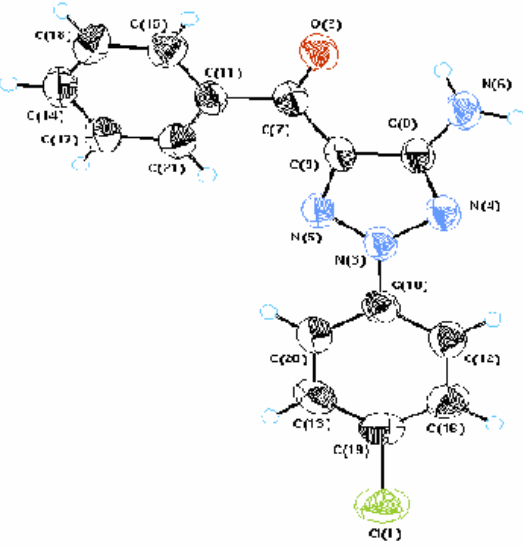

Figure 2. X-ray crystal structure of compound 5g.

It was found also that on refluxing triazole $\mathbf{5} \mathbf{f}$ in acetic anhydride it gave the acetylated product 8. This same product was obtained via refluxing $\mathbf{4 f}$ in acetic anhydride for long time, where $\mathbf{4 f}$ is firstly acetylated to 1,2,4-oxadiazole intermediate $\mathbf{7}$ which readily rearranges into the 
acetyl product 8 . The triazole $\mathbf{5 f}$ also condensed with malononitrile yielding $\mathbf{1 0}$ most likely via intermediate 9.

Table 1. Bond lengths and bind angles of compound $5 \mathbf{g}$

\begin{tabular}{cclc}
\hline Bond & Bond length & Bond & Bond angle \\
\hline N3-N4 & 1.358 & N3-N4-C8 & 102.80 \\
N3-N5 & 1.324 & N3-N5-C9 & 104.0 \\
N3-C10 & 1.416 & N4-N3-N5 & 115.88 \\
N4-C8 & 1.338 & N4-N3-C10 & 122.5 \\
N6-C8 & 1.356 & N4-C8-N6 & 122.2 \\
N5-C9 & 1.340 & N5-N3-C10 & 121.6 \\
C8-C9 & 1.417 & N5-C9-C8 & 108.3 \\
\hline
\end{tabular}

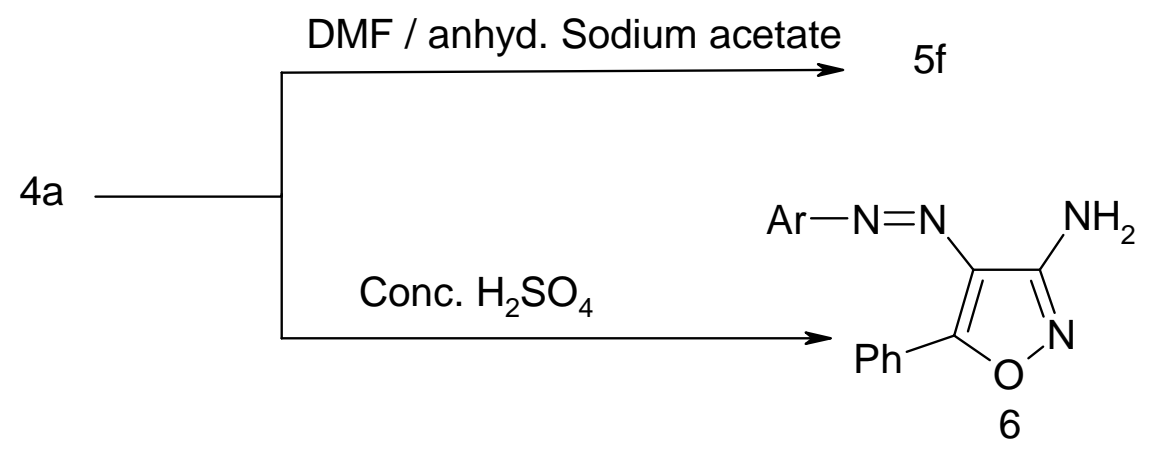

Scheme 3

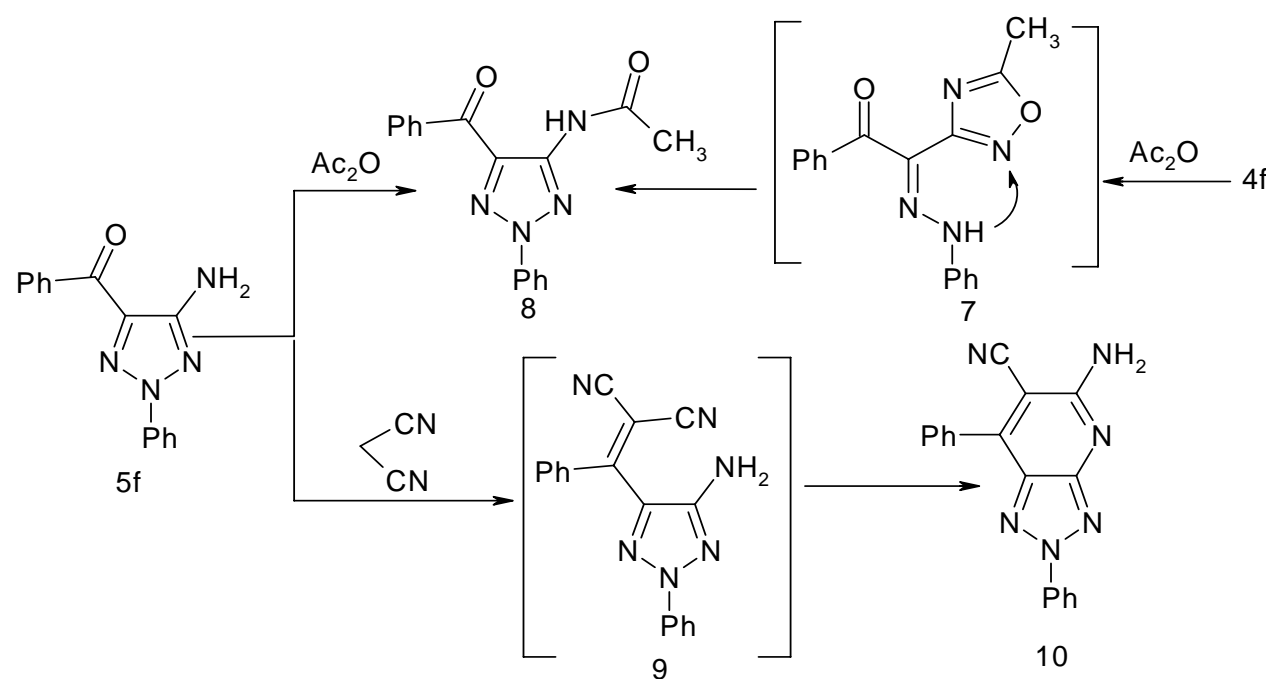

Scheme 4 


\section{Conclusions}

In conclusion a new simple approach to 1,2.3-triazoles amine from 2-arylhydrazononitriles have been achieved. Utility of triazole amines for synthesis of triazolo[4,5-b]pyridine-6-carbonitrile is also achieved.

\section{Experimental}

General Procedures. Melting points were determined on a Stuart melting point apparatus and are uncorrected. The IR spectra were recorded in $\mathrm{KBr}$ using a FTIR unit Bruker-vector 22 spectrophotometer. The ${ }^{1} \mathrm{H}$ and ${ }^{13} \mathrm{CNMR}$ spectra were recorded in DMSO- $\mathrm{d}_{6}$ as solvent at 300 MHz on Varian Gemini NMR spectrometer using TMS as internal standard. Chemical shifts are reported in $\delta$ units (ppm). Mass spectra were measured on a Shimadzu GMMS -QP-1000 EX mass spectrometer at $70 \mathrm{eV}$. The crystal structure was determined by the $\mathrm{X}$-ray unit at the National Research Center, Dokki, Cairo.

Crystallographic analysis for compound 5g. The crystals were mounted on a glass fiber. All measurements were performed on an ENRAF NONIUNS FR 590. The data were collected at temperature $20 \pm 1{ }^{\circ} \mathrm{C}$ using the $\omega$ scanning technique to a maximum of 20 of $27.12^{\circ}$. The temperature was solved by direct method using SIR 92 and full-matrix least squares. Non hydrogen atoms were refined anisotropically. Hydrogen atoms were located geometrically and were refined isotropically.

\section{Crystal data}

$\mathrm{C}_{15} \mathrm{H}_{11} \mathrm{~N}_{4} \mathrm{O}, \mathrm{M}=298.733$, monoclinic, $a=12.1876$ (4), $b=7.8561$ (2), $\mathrm{c}=17.7250$ (7) $\AA, \alpha=\gamma=$ $90.00^{\circ}, \beta=13 .(18) * 10^{10}$ space group: $\mathrm{P} 2_{1} / \mathrm{c} . \mathrm{Z}=4, \mathrm{D}_{\mathrm{x}}=1.426 \mathrm{Mg} \mathrm{m}^{-3}$, reflection 5739 measured, $\theta_{\max }=27.50^{\circ}, \omega \mathrm{R}$ factor $=0.097$

\section{General procedures for compounds $4 \mathrm{a}-\mathrm{g}$}

A mixture of arylhydrazononitriles $2(10 \mathrm{mmol})$, and hydroxyl amine was refluxed in ethanol (20 $\mathrm{ml}$ ) in presence of sodiumacetate for $2 \mathrm{~h}$. The solvent was evaporated under vacuum and the crude product was collected and crystallized from ethanol.

2-(1H-Benzoimidazol-2-yl)- $N$-hydroxy-2-(phenyl-hydrazono)-acetamidine (4a). Yield: 2.23 g (76\%), mp: $232{ }^{\circ} \mathrm{C}$. Anal. Calcd. for $\mathrm{C}_{15} \mathrm{H}_{14} \mathrm{~N}_{6} \mathrm{O}$ (294.32): C, 61.22; H, 4.79; N, 28.55. Found: $\mathrm{C}, 61.34 ; \mathrm{H}, 4.74 ; \mathrm{N}, 28.64$. IR $\left(\mathrm{KBr}, \mathrm{cm}^{-1}\right): 3455.9,3358.5,3255.2\left(\mathrm{NH}_{2}, \mathrm{NH}, \mathrm{OH}\right) ;{ }^{1} \mathrm{H} \mathrm{MNR}$ (300 MHz, DMSO-d6): $\delta$, ppm 5.89 (s, 2H, NH ), 6.96-7.84 (m, 9H, Ar-H) 9.98 (s, 1H, OH), 12.42 (s, 1H, benzimidazole-NH), $14.33(\mathrm{~s}, 1 \mathrm{H}, \mathrm{NH}) ;{ }^{13} \mathrm{C}$ NMR (300 MHz, DMSO-d6) : $\delta, \mathrm{ppm}$ 112.47, 113.93, 118.77, 119.15, 121.68, 122.36, 123.94, 129.33, 131.56, 140.96, 143.28, 145.64, 150.69; MS (EI): $\mathrm{m} / \mathrm{z}(\%)=294\left(\mathrm{M}^{+}\right)$.

2-Benzothiazol-2-yl- $\boldsymbol{N}$-hydroxy-2-(phenyl-hydrazono)-acetamidine (4c). Yield: $2.39 \mathrm{~g} \mathrm{(77 \% ),}$ mp: $168-170{ }^{\circ} \mathrm{C}$. Anal. Calcd. for $\mathrm{C}_{15} \mathrm{H}_{13} \mathrm{~N}_{5} \mathrm{OS}$ (311.37): C, 57.86; H, 4.21; N, 22.49. Found: $\mathrm{C}$, 
57.92; H, 4.32; N, 22.38. IR (KBr, $\left.\mathrm{cm}^{-1}\right): 3445.6,324.6,3054.2\left(\mathrm{NH}_{2}, \mathrm{NH}, \mathrm{OH}\right) ;{ }^{1} \mathrm{H}$ MNR $(300$ MHz, DMSO-d6) : $\delta$, ppm $5.80\left(\mathrm{~s}, 1 \mathrm{H}, \mathrm{NH}_{2}\right), 7.26$ (br s, $\left.1 \mathrm{H}, \mathrm{NH}_{2}\right), 7.04-8.30(\mathrm{~m}, 9 \mathrm{H}, \mathrm{Ar}-\mathrm{H})$, 10.09 (s, $0.46 \mathrm{H}, \mathrm{OH}), 10.43$ (s, $0.54 \mathrm{H}, \mathrm{OH}), 13.58$ (s, $0.56 \mathrm{H}, \mathrm{NH}), 14.48$ (s, $0.44 \mathrm{H}, \mathrm{NH})$; MS (EI): $\mathrm{m} / \mathrm{z}(\%)=311\left(\mathrm{M}^{+}\right)$.

2-Benzothiazol-2-yl) -2-[(4-chloro-phenyl)-hydrazono)]- $\boldsymbol{N}$-hydroxy-acetamidine (4d). Yield: 2.70 g (78\%), mp: $299-201{ }^{\circ} \mathrm{C}$. Anal. Calcd. for $\mathrm{C}_{15} \mathrm{H}_{12} \mathrm{ClN}_{5} \mathrm{OS}$ (345.81): C, 52.10; H, 3.50; N, 20.25. Found: C, 52.23; H, 3.41; N, 20.33. IR $\left(\mathrm{KBr}, \mathrm{cm}^{-1}\right): 3425.4,3354.3,3266.5,3057.4\left(\mathrm{NH}_{2}\right.$, $\mathrm{NH}, \mathrm{OH}$ ); ${ }^{1} \mathrm{H}$ MNR (300 MHz, DMSO-d6) : $\delta$, ppm 5.83 (s, $1 \mathrm{H}, \mathrm{NH}_{2}$ ), 7.27 (br s, $1 \mathrm{H}, \mathrm{NH}_{2}$ ), 7.30-8.32 (m, 8H, Ar-H), 10.12 (s, 0.52 H, OH), 10.44 (s, 0.48 H, OH), 13.57 (s, 0.48 H, NH), $14.48(\mathrm{~s}, 0.52 \mathrm{H}, \mathrm{NH}) ; \mathrm{MS}(\mathrm{EI}): \mathrm{m} / \mathrm{z}(\%)=345\left(\mathrm{M}^{+}\right)$.

\section{General procedures for compounds $5 \mathrm{a}-\mathrm{g}$}

A mixture of arylhydrazononitriles $2(10 \mathrm{mmol})$, and hydroxyl amine was refluxed in DMF (20 $\mathrm{ml})$ in presence of anhydrous sodiumacetate $(2 \mathrm{~g})$ for $8 \mathrm{~h}$. The solvent was evaporated under vacuum and the crude product was collected and crystallized from proper solvent.

5-(1H-Benzoimidazol-2-yl)-2-phenyl-2H-[1,2,3]triazol-4-ylamine (5a). Yield: $2.24 \mathrm{~g} \mathrm{(81 \% ),}$ mp: $248-250{ }^{\circ} \mathrm{C}$. Anal. Calcd. for $\mathrm{C}_{15} \mathrm{H}_{12} \mathrm{~N}_{6}$ (276.30): C, 65.21; H, 4.38; N, 30.42. Found: C, 65.37; H, 4.29; N, 30.53. IR (KBr, cm $\left.{ }^{-1}\right): 336.3,3278.8,3151.5\left(\mathrm{NH}_{2}, \mathrm{NH}\right) ;{ }^{1} \mathrm{H}$ MNR $(300 \mathrm{MHz}$, DMSO-d6): $\delta$, ppm 6.32 (s, 2H, NH ${ }_{2}$ ), 7.23 - 7.98 (m, 9H, Ar-H), 13.05 (s, 1H, NH); MS (EI): $\mathrm{m} / \mathrm{z}(\%)=276\left(\mathrm{M}^{+}\right)$.

5-(1H-Benzoimidazol-2-yl)-2-(4-chloro-phenyl-2H-[1,2,3]triazol-4-ylamine (5b). Yield: 2.36 g (76\%), mp: 278-280 ${ }^{\circ} \mathrm{C}$. Anal. Calcd. for $\mathrm{C}_{15} \mathrm{H}_{11} \mathrm{ClN}_{6}$ (310.75): C, 57.98; H, 3.57; N, 27.04. Found: C, 57.86; H, 3.42; N, 27.13. IR $\left(\mathrm{KBr}, \mathrm{cm}^{-1}\right): 3360.8,3273.1,3191.2\left(\mathrm{NH}_{2}, \mathrm{NH}\right) ;{ }^{1} \mathrm{H}$ MNR (300 MHz, DMSO-d6) : $\delta$, ppm $6.38\left(\mathrm{~s}, 2 \mathrm{H}, \mathrm{NH}_{2}\right), 7.25(\mathrm{~d}, 2 \mathrm{H}, \mathrm{Ar}-\mathrm{H}), 7.55-7.65(\mathrm{~m}, 4 \mathrm{H}$, benzimidazole-H), $7.96(\mathrm{~d}, 2 \mathrm{H}, \mathrm{Ar}-\mathrm{H}), 13.08(\mathrm{~s}, 1 \mathrm{H}$, benzimidazole-NH); MS (EI): $\mathrm{m} / \mathrm{z}(\%)=$ $310\left(\mathrm{M}^{+}\right)$.

5-Benzothiazol-2-yl)-2-phenyl-2H-[1,2,3]triazol-4-ylamine (5c). Yield: $2.17 \mathrm{~g}$ (74\%), mp: 205-207 ${ }^{\circ} \mathrm{C}$. Anal. Calcd. for $\mathrm{C}_{15} \mathrm{H}_{11} \mathrm{~N}_{5} \mathrm{~S}$ (293.35): C, 61.42; H, 3.78; N, 23.87. Found: C, 61.53; $\mathrm{H}, 371 ; \mathrm{N}, 23.96$. IR (KBr, cm $\left.{ }^{-1}\right): v$ 3401.4, $3294.4\left(\mathrm{NH}_{2}\right) ;{ }^{1} \mathrm{H}$ MNR (300 MHz, DMSO-d6) : $\delta$, ppm 6.45 (s, 2H, $\left.\mathrm{NH}_{2}\right), 7.37-7.57$ (m, 5H, Ph-H), 7.93-8.14 (m, 4H, benzothiazol-H); MS (EI): $\mathrm{m} / \mathrm{z}(\%)=293\left(\mathrm{M}^{+}\right)$.

5-Benzothiazol-2-yl)-2-(4-chloro-phenyl)-2H-[1,2,3]triazol-4-ylamine (5d). Yield: $2.56 \mathrm{~g}$ (78\%), mp: 230-232 ${ }^{\circ} \mathrm{C}$. Anal. Calcd. for $\mathrm{C}_{15} \mathrm{H}_{10} \mathrm{ClN}_{5} \mathrm{~S}$ (327.80): C, 54.96; H, 3.07; N, 21.36 . Found: C, 54.89; H, 3.18; N, 21.42. IR $\left(\mathrm{KBr}, \mathrm{cm}^{-1}\right): 3303.7,3150.4\left(\mathrm{NH}_{2}\right) ;{ }^{1} \mathrm{H}$ MNR $(300 \mathrm{MHz}$, DMSO-d6) : $\delta$, ppm 6.91 (s, 2H, NH ${ }_{2}$ ), 7.46-8.16 (m, 8H, Ar-H); ${ }^{13} \mathrm{C}$ NMR (DMSO-d6) : $\delta$, ppm 122.26, 123.18, 125.49, 125.81, 126.54, 129.46, 132.14, 134.46, 135.45, 153.42, 154.27, 155.77, 159.17; MS (EI): $\mathrm{m} / \mathrm{z}(\%)=327\left(\mathrm{M}^{+}\right)$.

2-(4-Chlorophenyl)-5-phenyl-2H-[1,2,3]triazole-4-ylamine (5e). Yield: 2.19 g (81\%), mp: $165^{\circ} \mathrm{C}$. Anal. Calcd. for $\mathrm{C}_{14} \mathrm{H}_{11} \mathrm{ClN}_{4}$ (270.72): C, 62.11; H, 4.10; N, 20.71. Found: C, 62.31; H, 4.12; N, 20.83. IR (KBr, cm $\left.{ }^{-1}\right): 3331.3,3170.4\left(\mathrm{NH}_{2}\right) ;{ }^{1} \mathrm{H}$ MNR $(300 \mathrm{MHz}$, DMSO-d6) : $\delta$, ppm $5.86\left(\mathrm{~s}, 2 \mathrm{H}, \mathrm{NH}_{2}\right), 7.36-7.78(\mathrm{~m}, 9 \mathrm{H}, \mathrm{Ar}-\mathrm{H})$; $\mathrm{MS}(\mathrm{EI}): \mathrm{m} / \mathrm{z}(\%)=270\left(\mathrm{M}^{+}\right)$. 
5-Amino-2-phenyl-2H-[1,2,3]triazole-4-yl)-phenyl-methanone (5f). Yield: $1.95 \mathrm{~g}$ (74\%), mp: 102-103 ${ }^{\circ} \mathrm{C}$. Anal. Calcd. for $\mathrm{C}_{15} \mathrm{H}_{12} \mathrm{~N}_{4} \mathrm{O}$ (264.29): C, 68.17; H, 4.58; N, 21.20. Found: C, 68.23; $\mathrm{H}, 4.38$; N, 21.26. IR ( $\left.\mathrm{KBr}, \mathrm{cm}^{-1}\right)$ : 3426.9, $3295.1\left(\mathrm{NH}_{2}\right), 1632.5(\mathrm{CO}) ;{ }^{1} \mathrm{H}$ MNR (300 MHz, DMSO-d6) : $\delta$, ppm $6.56\left(\mathrm{~s}, 2 \mathrm{H}, \mathrm{NH}_{2}\right), 7.38-8.32(\mathrm{~m}, 10 \mathrm{H}, \mathrm{Ar}-\mathrm{H}) ;{ }^{13} \mathrm{C}$ NMR $(300 \mathrm{MHz}, \mathrm{DMSO}-$ d6) : $\delta$, ppm 118.39, 127.83, 128.38, 129.58, 129.62, 130.84, 132.76, 136.87, 138.71, 156.71, 185.98; MS (EI): $\mathrm{m} / \mathrm{z}(\%)=264\left(\mathrm{M}^{+}\right)$.

5-Amino-2-(4-chloro-phenyl)-2H-[1,2,3]triazol-4-yl]-phenyl-methanone (5g). Yield: $2.24 \mathrm{~g}$ (75\%), mp: $158-160{ }^{\circ} \mathrm{C}$. Anal. Calcd. for $\mathrm{C}_{15} \mathrm{H}_{11} \mathrm{ClN}_{4} \mathrm{O}$ (298.73): C, 60.31; H, 3.71; N, 18.75 . Found: C, 60.43; H, 3.67; N, 18.69. IR (KBr, cm $\left.{ }^{-1}\right): 3458.1,3352.7\left(\mathrm{NH}_{2}\right), 1627.8(\mathrm{CO}) ;{ }^{1} \mathrm{H}$ MNR (300 MHz, DMSO-d6): $\delta$, ppm 6.58 (s, 2H, NH 2 ), 7.55 - 8.29 (m, 9H, Ar-H); MS (EI): $\mathrm{m} / \mathrm{z}(\%)=298\left(\mathrm{M}^{+}\right)$.

\section{Preparation of $\mathrm{N}$-(5-Benzoyl-2-phenyl-2H-[1,2,3]azol-4-yl)acetamide (8)}

Method A. $10 \mathrm{mmol}$ of triazole $5 \mathrm{f}$ was refluxed in acetic anhydride $(20 \mathrm{ml})$ for $1.5 \mathrm{~h}$. The solvent was evaporated under vacuum and the crude product was collected and crystallized from ethanol.

Method B. $10 \mathrm{mmol}$ of amidoxime $4 \mathrm{f}$ was refluxed in acetic anhydride $(20 \mathrm{ml})$ for $4 \mathrm{~h}$. The solvent was evaporated under vacuum and the crude product was collected and crystallized from ethanol.

Yield: $2.27 \mathrm{~g}$ (74\%), mp: 138-140 ${ }^{\circ} \mathrm{C}$. Anal. Calcd. for $\mathrm{C}_{17} \mathrm{H}_{14} \mathrm{~N}_{4} \mathrm{O}_{2}$ (306.33): C, 66.66; H, 4.61; $\mathrm{N}, 18.29$. Found: C, 66.42; H, 4.73; N, 18.26. IR (KBr, $\left.\mathrm{cm}^{-1}\right): 3324.5(\mathrm{NH}), 1692.8,1636.7$ (CO); ${ }^{1} \mathrm{H}$ MNR (300 MHz, DMSO-d6): $\delta$, ppm 2.07 (s, 3H, $\mathrm{CH}_{3}$ ), 7.43-8.08 (m, 10H, Ar-H), 10.71 (s, 1H, NH); ${ }^{13} \mathrm{C}$ NMR (DMSO-d6): $\delta$, ppm 22.81, 118.56, 128.26, 128.47, 129.64, $129.69,133.40,136.32,137.26,138.58,144.85,168.05,185.99 ; \mathrm{MS}(\mathrm{EI}): \mathrm{m} / \mathrm{z}(\%)=306\left(\mathrm{M}^{+}\right)$.

Preparation of 5-Amino-2,7-diphenyl-2H-[1,2,3]triazolo[4,5-b]pyridine-6-carbonitrile (10) A mixture of triazole $5 \mathbf{f}(10 \mathrm{mmol})$, and malononitrile $(10 \mathrm{mmol})$ was refluxed in ethanol $(20 \mathrm{ml})$ in presence of piperidine $(0.5 \mathrm{ml})$ for $4 \mathrm{~h}$. The solvent was evaporated under vacuum and the crude product was collected and crystallized from ethanol.

Yield: 2.12 g (68\%), mp: $285-287^{\circ} \mathrm{C}$. Anal. Calcd. for $\mathrm{C}_{18} \mathrm{H}_{12} \mathrm{~N}_{6}(312.34)$ : C, 69.22; H, 3.87; N, 26.91. Found: C, 69.31; H, 3.84; N, 27.01. IR ( $\left.\mathrm{KBr}, \mathrm{cm}^{-1}\right): 3409.5,3333.3\left(\mathrm{NH}_{2}\right), 2213.9(\mathrm{CN})$; ${ }^{1} \mathrm{H}$ MNR (300 MHz, DMSO-d6): $\delta$, ppm 4.10 (s, 2H, NH ), 7.28-7.46 (m, 10H, ph-H); MS (EI): $\mathrm{m} / \mathrm{z}(\%)=312\left(\mathrm{M}^{+}\right)$.

\section{References}

1. Kadaba, P. U. J. Med. Chem. 1988, 31, 196.

2. Wamhoff, H. In Comprehensive Heterocyclic Chemistry, K. T. Potts, Ed., Pergamon: Oxford, 1984; pp 669.

3. Ferrarini, P. L.; Livi, O. Il Farmaco ed. Sci. 1981, 83, 243. 
4. Matloubi, H.; Shafiee, A.; Saemian, N.; Shirvani, G.; Daha, F. J., Applied Radiation and Isotopes 2004, 60, 665.

5. Invidiata, F. Paolo; Aiello, S.; Furno, G.; Aiello, E. J. Heterocycl. Chem. 2000, 30, 355.

6. Lalezari, I.; Gomez, L. A.; Khorshidi, M. J. Heterocycl. Chem. 1990, 27, 687.

7. Molteni, G.; Del Buttero, P. Tetrahedron 2005, 61, 4983.

8. Zhao, Y.-B.; Yan, Z.-Y.; Liang, Y.-M. Tetrahedron Lett. 2006, 47, 1545.

9. Chittaboina, S.; Xie, F.; Wang, Q. Tetrahedron Lett. 2005, 46, 2331.

10. Kiselyou, A. S. Tetrahedron Lett. 2006, 47, 2631.

11. Batori, S.; Gacs-Baitz, E.; Bokotey, S.; Messmer, A. Tetrahedron 2003, 59, 4297.

12. IJsselstijn, M.; Cintrat, J.-C. Tetrahedron, Science direct online, Click chemistry with ynamides.

13. Huisgen, R.; Knorr, R.; Mobius, L.; Szeimies, Chem. Ber 1965, 98, 4014.

14. Businelli, S.; Martino, E. D.; Zanirato, P. Arkivoc 2001, (i), 131.

15. Fan, W. Q.; Katritzky, A. R. Comprehensive Heterocyclic Chemistry II, Elsevier: New York, 1996; Vol 4.

16. Rai, B; Lakhan, R. Indian J. Chem., Sect. B 1989, $28 B(7), 595$.

17. Swartz, D. L.; Karash, A. R.; Berry, L. A. and Jaeger, D. L. Journal of Heterocycl. Chem. 1983, 20, 1561.

18. Elnagdi, N. M., Ph.D. Thesis, Cairo University, 2006.

19. Elnagdi, M. H.; Elmoghayer, M. R. H.; Hafez, E. A.; Almina, H. H. J. Org. Chem. 1975, 40, 2604.

20. Further details of the crystal structure of $\mathbf{5 g}$ will be available in the Cambridge Crystallographic Data center under deposit nos. CCDC 613081. 